\title{
BENEFITS OF NURSE PRESCRIBING FOR PATIENTS IN PAIN: NURSES' VIEWS
}

Karen Stenner BSc (Hons), Research Fellow, School of Health and Social Care, University of Reading, UK. Email: k.l.stenner@ reading.ac.uk

Molly Courtenay, MSc, PhD, RN, Professor of Prescribing and Medicines

Management, University of Reading.

Short Title: Benefits of Nurse Prescribing in Pain

This paper has been published as: Stenner K., \& Courtenay M. (2008) Benefits of nurse prescribing for patients in pain: nurse's views. Journal of Advanced Nursing, 63 (1), 27-35.

The definite version is available at: www.blackwell-synergy.com 


\section{ABSTRACT}

\section{Aim}

This paper is a report of a study exploring nurses' views on the benefits of adopting the role of prescribing for patients with acute and chronic pain.

\section{Background}

It was envisioned that the advent of nurse prescribing would be beneficial to the efficiency and effectiveness of the United Kingdom National Health Service. Research to date does indeed indicate that nurse prescribing can be beneficial to patients, nurses and the health service in general. Despite the expansion of nurse prescribing, there is little evidence of its impact according to nurses working in specialist areas, such as with patients in acute and chronic pain.

Method: Interviews were conducted during 2006 and 2007 with 26 nurses qualified to prescribe medicines for patients in acute and chronic pain. This was a qualitative study and a thematic analysis was conducted.

Findings: Nurses reported a number of benefits, including faster access to treatment, improved quality of care, more appropriate prescribing of medication, improved safety, improved relations and communication with patients, greater efficiency and cost effectiveness. Nurses benefited from increased job satisfaction, credibility with patients and healthcare professionals and also gained knowledge through prescribing.

Conclusion: There is potential for the benefits of nurse prescribing to be expanded beyond the United Kingdom in settings where nurses hold similar roles in the treatment 
of pain, although further research is recommended to substantiate these findings using a wider range of research methods. 


\section{Keywords}

Nurse prescribing, pain management, qualitative research, interviews, Controlled Drugs

\section{SUMMARY}

\section{What is already known about this topic}

- A number of benefits of nurse prescribing have been identified, including improved patient care, time-saving, increased job satisfaction and greater autonomy for nurses

- A nursing approach to patient consultation and information-giving is thought to result in more holistic patient care during prescribing

- There is little known about the impact of nurse prescribing for patients with acute and chronic pain

\section{What this paper adds}

- Nurses prescribing for patients in pain report similar benefits to those reported by nurses working in other therapeutic areas

- Amongst other benefits, specialist pain nurses report improvements in appropriate choice of medication, safety, quality of care, efficiency and cost effectiveness, arising from nurse prescribing

- Autonomy, specialist knowledge and experience, and enhanced consultation skills are reported to be central to the benefits of nurse prescribing for patients in pain 


\section{INTRODUCTION}

The role of nurses in prescribing medicines is continually evolving both in the United Kingdom (UK) and internationally. In the UK, nurse prescribing was born out of the need to increase efficiency in the National Health Service (NHS) by making best use of its resources. Nurse-led services are one means of improving healthcare provision (Department of Health (DoH) 1999a, 2000), and a string of legislative change has gradually broadened the scope of nurse prescribing in the UK (Courtenay and Carey 2007).

In the UK, appropriately-qualified community nurses are able to assess, diagnose and prescribe independently from a limited list of medicines included in the Nurse Prescribers' Formulary for Community Practitioners. Nurse Independent Prescribers (NIPs) (previously known as independent extended prescribers) are similarly able to independently assess, diagnose and prescribe any licensed medicine and some Controlled Drugs (CDs) that is drugs listed in the schedule to the Narcotics Control Regulations of the Controlled Drugs Act, including barbiturates and many opiates (DoH 2005). By contrast, Nurse Supplementary Prescribing (DoH 2002) takes place after assessment and diagnosis of a patient's condition has been made by a doctor, and a Clinical Management Plan (CMP) has been drawn up for the patient. The CMP includes a list of medicines from which the supplementary prescriber is able to prescribe. Nurse Supplementary Prescribers (NSPs) are able to prescribe any medicine (including unlicensed medicines and CDs), and this mode of prescribing is best suited to patients with long-term chronic conditions. Training for Nurse Independent and Nurse Supplementary Prescribing is 
combined, i.e. nurses successfully completing the prescribing programme are awarded the dual qualification of NIP/NSP. There are now over 10,000 nurses in the UK able to prescribe both as NIPs and NSPs (Nursing and Midwifery Council 2007).

The introduction of prescribing is a recent addition to the role of the pain nurse (Stenner and Courtenay 2007). Specialist pain nurses play a key role across a variety of settings and their role in the safe management of acute and chronic pain has been emphasized (Royal College of Anesthetists and the Pain Society 2003). There are specialist pain services operating in inpatient and outpatient clinics for acute and chronic pain within hospital settings and in primary care facilities. There are also oncology and palliative care units within hospitals and on external sites.

Whilst there have been a number of studies of the impact of nurse prescribing, there is a lack of research on nurse prescribing of medicines for acute and chronic pain (including palliative care settings). Given this lack of information, a qualitative design was chosen that would enable us to explore the implementation of nurse prescribing from the point of view of nurses.

\section{BACKGROUND}

The potential benefits of nurse prescribing, as set out in the Crown Report (DoH 1999b), include: improved use of time on behalf of patients and professionals, improved patient care, best use of appropriate skills and improved communication between teams. Similar 
benefits were anticipated in the guidance on implementing independent prescribing (DoH 2006):

- Improved patient care without compromising patient safety

- Making it easier for patients to get the medicines they need

- Increased patient choice in accessing medicines

- Better use of the skills of health professionals

- Contributing to the introduction of more flexible team working across the NHS

Some of these benefits have been substantiated through research. The nature of the nursepatient consultation is a key feature in research on improving patient care. It has been reported that patients believe that nurses are more approachable, informative and can be more knowledgeable about specific areas than doctors (Luker et al 1997, 1998, Brooks et al 2001). Nurses believe that they give more advice to patients about their condition and treatment than doctors (Luker and McHugh 2002), and some doctors share this opinion (Avery et al 2004). There are indications that prescribing has enabled nurses to achieve more holistic patient assessment, resulting in more appropriate treatment (Luker and McHugh 2002, Lewis-Evans and Jester 2004). Bradley and Nolan (2007), in a recent qualitative study involving interviews with 45 nurse prescribers in the UK, found that nurses believed that prescribing complemented their nursing practice, allowing for more holistic practice and greater opportunity for patient involvement.

As regards the safety of nurse prescribing, patients and doctors believe that nurses are aware of their own limitations and encourage safe practice (Brooks et al 2001, Avery et 
al 2004). To provide a measure of the quality and safety of nurses' independent prescribing practices, observational case studies were conducted by Latter et al (2007a). These researchers found that nurses wrote accurate prescriptions, although there were inconsistencies in the application of assessment and diagnostic techniques and detailed recordings in patient notes.

Increased patient choice in accessing medicines can occur either by increasing the available routes by which patients can access health care or by increasing patient choice over treatment options. Whilst it is reported that nurses are prescribing appropriate medicine for patients, there is little evidence for improved patient involvement in that choice (Latter et al 2007b). However, improvements have been reported in the timeliness and convenience of patient access to medicines (Luker et al, 1998, Brooks et al 2001, Luker and McHugh 2002, Latter and Courtenay 2004, Lewis-Evans and Jester 2004). Increased nurse autonomy and reduced dependency on doctors (Rodden 2001, Bradley and Nolan 2007), may in turn, help to improve the scope and efficiency of nurse-led services.

There are indications that nurse prescribing makes better use of nurses' and doctors' time and skills, increases nurse job satisfaction and improves professional relationships (Avery et al 2004, Latter et al 2005, Bradley and Nolan 2007). Increased autonomy and decreased dependence on doctors by nurse prescribers also has a postivie impact on team relationships (Rodden 2001, Bradley and Nolan 2007). 
A substantial amount of the early research focused on the impact of prescribing in relation to community nurses (district nurses and health visitors). Other research has reported anticipated benefits to areas where nurses were not yet prescribing (Nolan et al 2001, Hay et al 2004, Bradley et al 2005, Pontin and Jones 2007). Research specific to nurse prescribing for patients in pain is lacking, although there is some evidence about the role of pain nurses in general. A survey of over 3000 nurses working with patients with acute and chronic pain in hospital settings in the United States of America showed that nurses were involved in assessment, monitoring, evaluating and pain management (Pellino et al 2002). Data collected from 100 Advanced Nurse Practitioners in Canada by questionnaire (Kohr and Sawhney 2005) and interview data from 15 nurses working in the post-operative pain setting in Sweden (Soderhamn and Idvall 2003) indicate that nurses also play an important role in advising and influencing medical decisions about prescribing for pain. In the UK, a questionnaire survey of 103 nurse members of the Pain Society was conducted to explore nurses' beliefs about pain management and their understanding of the roles of members of the pain team (Brown and Richardson 2006). Interdisciplinary team working and collaboration were identified as central to the role of these nurses. Similar findings were reported by Johnston and Smith (2006) in a qualitative study exploring the concept of palliative care and the role of palliative care nurses. They conducted 44 interviews with nurses and patients working in acute and hospice settings in Scotland and found that a cohesive multidisciplinary team was considered to be a key factor in effective palliative care nursing. 
No evidence is currently available on pain nurses' views on the adoption of prescribing in their practice. This is important as there is a continual need for research to inform and shape this area of work as it expands to different areas of nursing practice. At the time of writing, views were being sought on proposals to expand the range of Controlled Drugs that can be prescribed by Nurse Independent Prescribers (Home Office 2007). It is therefore important to understand the role that nurses play in prescribing for patients in pain.

\section{THE STUDY}

\section{Aim}

The study aim was to explore nurses' views on adopting the role of prescribing for patients with acute and chronic pain.

Findings relating to further study objectives are reported elsewhere (Stenner and Courtenay 2007).

\section{Design}

A qualitative design was selected, using semi-structured interviews and thematic analysis. The data were collected in 2006-07.

\section{Participants}

The participants were purposively sampled from two nationally-recognised groups of specialist pain nurses in England. They were 26 nurses, qualified in NIP and NSP, who 
worked in acute and chronic (including palliative) pain services in a range of UK work settings, and included clinical nurse specialists in pain $(n=15)$, Macmillan nurses (charity funded cancer nurses) $(n=2)$, pain consultants $(n=6)$, clinical nurse managers $(n=2)$ and a lead pain nurse (i.e. a nurse working in this specialist area and responsible for leading and developing a nursing team). The majority of participants (19) worked in hospital inpatient and/or outpatient settings and treated mixed pain types (i.e. acute, chronic and palliative pain), two worked in a hospital outpatient department managing chronic pain and one in a walk-in-centre managing acute and chronic pain. A further 3 participants provided community home care and managed palliative pain, and one worked in a hospice and managed palliative pain.

\section{Data collection}

Audio-recorded, face-to-face, semi-structured interviews lasting 30-50 minutes were held in quiet locations convenient to participants. The interview schedule was informed by a review of the relevant literature and national work in the area of nurse prescribing (Courtenay and Carey 2007, Latter et al 2005). The interview schedule covered general views on nurse prescribing, role changes resulting from prescribing, difficulties in prescribing, enabling factors in prescribing, support and supervision, educational needs, views on NSP and views on the prescribing training course.

\section{Ethical considerations}

The study was approved by the appropriate university and NHS ethics and research governance committees. Information about the study was distributed to members of the 
two national pain groups and those interested in participating were recruited by the group organisers. Contact details were then passed to us and we provided further information to potential participants and answered queries about the project. Consent was obtained prior to interview and participants were free to withdraw from the study at any time. Transcripts were anonymised by removing identifying features.

\section{Data analysis}

Thematic data analysis was conducted. The process was data driven, or inductive, in that a coding frame was developed on the basis of the data rather than being pre-determined by theory (Patton 1990). Initial coding and identification of patterns across the data were aided by the use of the ATLAS.ti qualitative data analysis software package. A further process of interpreting the broader meanings and significance of the patterns in the data was used to identify overarching themes and sub-themes.

\section{Rigour}

The coding frame was independently assessed by an additional qualitative researcher. Saturation was achieved in that we were able to categorise the later interview data within the existing coding frame without needing to add new codes. The main findings were

presented to pain nurses at a pain network meeting in May 2007 and a nurse pain consultant meeting in June 2007. A high level of agreement and acknowledgement of the relevance of the findings were reported. 


\section{FINDINGS}

\section{Autonomy}

Autonomy, the sense of being free to make informed prescribing decisions, was the key concept behind a number of benefits to nurse prescribing in pain.

\section{$\underline{\text { Faster access to treatment }}$}

Improved speed and convenience of access to medicines for patients was reported as a key benefit. In acute settings, patients were reported to have regularly experienced delays in receiving medication advised by specialist pain nurses before the nurses took on the prescribing role. Greater autonomy over prescribing enabled nurses to overcome these delays by prescribing immediately and planning for faster access to medication:

"I would have advised the same medications that I now can prescribe, but the difference is that often I would advise something, go back the next day to see if what I had advised had worked to find that it had never been implemented. So you would be 24 hours down the line with no advance, whereas this way I can prescribe it, I can ensure that the pharmacist knows, it's up on the wards, it's ready, raring to go and we're 24 hours ahead of ourselves."

\section{Improved safety}

Misinterpretations had occurred previously, where nurses recommended medication but could not prescribe. Prescribing was believed to improve safety by ensuring that the person assessing the patient had responsibility for prescribing the medication advised. 
Importantly, a good knowledge base was considered necessary for prescribing CDs, and therefore any improvements in care were dependent upon the level of specialist knowledge and experience. Nurses described a process of demarcating those medicines they were competent and comfortable to prescribe. In some cases, drawing up an intent to prescribe' (listing the types of medication the nurse was able to prescribe), helped to formalize this process and was useful for explaining the limitations of practice to colleagues. Nurses in general were considered to be more cautious over safety in prescribing than junior medical doctors, who were seen to be regularly under pressure to prescribe outside of their area of competence:

"I feel it is a lot safer because we know the side-effects, we know the way that the drugs work - all my team have got a Master's in pain management so we understand the drugs and the pharmaco-kinetics and pharmaco-dynamics of it ... so we feel it has made it a lot safer because a lot of the medical colleagues don't understand the way that opioids work."

\section{Appropriate prescribing and quality of care}

Clinically-inappropriate prescribing of $\mathrm{CDs}$ had been noted by participants. Lack of experience and fear of prescribing CDs were the reasons given as to why some healthcare professionals prescribed inadequate doses. The most common was misjudging the appropriate strength of pain medication, which resulted in either over- or underprescribing. Other problems occurred in identifying contraindications and drug interactions, neglecting to take patients' views into account, making errors in drug calculations and sudden withdrawal of medication. 
Nurses reported that combining specialist skills, experience and knowledge of pain medication, together with a comprehensive patient assessment, enabled them to make more clinically-appropriate prescribing decisions. In other words, they reported an ability to choose products accurately and tailor medication to best suite a patient's condition, circumstances and medical history. Making the right choice of medication was believed to improve patients' trust in the service received, thus influencing concordance as well improving clinical and cost effectiveness:

"We see that a lot of the time with a lot of the junior doctors because they will under prescribe Oramorph ${ }^{\circledR}$, for instance, and then we come along and change the prescription and speak to them and say, 'Actually this dose, 5-10 milligrams, is completely inadequate - the dose is such and such'. So yes, there is definitely stigma attached to Controlled Drugs."

"Often when GPs initiate a controlled drug, say slow release morphine or a fentanyl patch, they will initiate it at quite a high dose and, of course, if they do that the patient is more at risk of getting side effects. And then you have lost the patient's confidence in that particular drug, even though it may be better for the patient at a lower dose. I think that is often an advantage - when we go in and review the patient and what previous analgesia they have had and the conversion rate to morphine or whatever drug - that you choose to get the dose right and maintain the patient's confidence in the drug." 
Furthermore, where nurses were able to follow up and re-evaluate patients, they said that they were better able to alter medication in response to patient need. Examples were given whereby such increased autonomy over medicines management had, according to the nurse, improved patient care as well as being cost-effective:

"You can be very responsive with patients and if you are titrating between [drugs], because I know that I can get back to see them in a week or ten days. I would make that appointment there and then. I would perhaps only prescribe them that number of tablets, or perhaps a few extra, so I think it is quite cost effective from the GP's point of view."

\section{$\underline{\text { Improved relations and communication with patients }}$}

Because prescribing enabled nurses to deliver a complete episode of care, this was believed to enhance the relationship between patient and nurse, thus having an impact on care. Increased autonomy meant that nurses could be more confident about when, what and how patients were medicated. As a result, nurses said that they were better able to communicate with patients about their medication, improving patient confidence and concordance:

"It's just fantastic now that you can say to patients, 'Just bear with me, I'll be a few minutes, we'll get this sorted out and we'll get on top of your pain'. Whereas 
before I didn't feel confident enough to be able to say that to the patients because I knew all the frustrations that I was going to have to be able to get that job done.”

The partnership between prescriber and patient was seen as an important factor in prescribing CDs. This involved discussing and agreeing a course of action with the patient in line with their medical history and past use of CDs. It was believed that patients gained a better understanding of their medication, the reasons for taking it and the correct regime for taking it when it was prescribed by a nurse. It was suggested that differences in training and longer appointment times meant that nurses held more detailed and comprehensive consultations with patients, including giving better advice and explanations and more often acting on patient preferences. These perceived differences between doctor and nurse consultations were thought to further enhance patient care when combined with nurse prescribing:

"I think because we have a longer period of time spent with the patient we have got more time to actually explain things in more detail and, you know, I think to ... we have better communication skills because of the training we have had in the subject. And, you know, it's as much about relationship with the patient as giving information."

$\underline{\text { Greater efficiency and cost-effectiveness }}$ 
In addition to time saving, increased efficiency resulted from developing a more seamless service and enhancing working relationships within teams. For those working in the community, autonomy in prescribing reduced the need to gain approval from large numbers of GPs, although nurses continued to communicate with GPs about medicines they had prescribed. By improving access to medication, some nurses believed they had prevented hospital admissions or speeded the recovery of patients to enable earlier discharge from hospital. Nurse prescribing resulted in fewer interruptions to the care process for patients and was expected to reduce waiting times.

"With the way that the NHS is going, with getting patients out of secondary care quicker, you know, it has been really highlighted to me that the acute pain team and their nurse prescribing skills is paramount to that. Because if patients are in pain they can't do their physiotherapy, they can't go home, and getting patients home fairly quickly so they don't get infections etc. is really important, and that's been highlighted by management".

Nurses working in hospitals reported variations in the prescribing practice of different clinical teams and junior doctors. Through promotion of evidence-based medicine, nurses believed that they introduced greater consistency in prescribing practice. More appropriate evidence-based choice of medication by nurse prescribers was believed to result in less drug wastage, lower cost and better concordance. On the whole, nurses were aware of the cost implications of different drugs, particularly those who were involved in local policy or decision-making. Nurses reported that, where evidence showed more than 
one drug to be effective, they would prescribe cheaper drugs as the first choice unless there was a good rationale for prescribing a more expensive drug, for example if the patient was opiate-resistant or unable to take medication in the usual way:

“Cost is important. I wouldn't hesitate to use an expensive opioid such as oxycodone with a patient who had already had a bad experience on morphine, but I would on someone who hadn't. So I'd start with the cheap and simple and then work my way through to the more expensive and 'ritzy', as it were. So cost does... it's that pyramid of prescribing - you want something safe and effective but it's also got to be cost-effective as well."

\section{$\underline{\text { Increased job satisfaction }}$}

Increased job satisfaction was consistently reported as an outcome of gaining autonomy and being able to complete episodes of care. It was also linked to the ability to overcome previous frustrations with the healthcare system and to make use of existing knowledge and skills, and to gaining confidence, witnessing positive outcomes and providing a more rounded quality care for patients:

"We wonder how we ever managed beforehand, because it gives us satisfaction because we're doing the holistic care. We have always assessed our patients and made a diagnosis, and now we can prescribe and then we can actually follow it up.” 


\section{Gaining knowledge through prescribing}

Prescribing did more than simply enable nurses to make better use of existing knowledge - it was also said to facilitate new learning. Nurses were continually learning from the process of prescribing, particularly where they were able to re-evaluate medications at follow-up sessions. Examples included learning about the effects of medication for a range of different patients, increasing knowledge of side effects and drug reactions, and increased knowledge of symptom control. Regular supervision time to review patient outcomes and discuss medicine interactions was reported to support this continual learning process:

"I think that when we are prescribing it gives us continuity of knowledge in that you know what the patients had because you prescribed it... I have learnt from the effects of drugs. Having taken the responsibility, I have learnt more about how that drug affects the patient, whereas before perhaps I wasn't as aware and maybe didn't pay it as much attention."

The act of prescribing itself encouraged continuous learning about drug interactions and side effects. Participants commonly described becoming more cautious and vigilant once they had passed the prescribing qualification and taken on direct responsibility for prescribing. This 'transition effect' from novice to expert was accompanied by systematic reviewing of patient notes, checking for contraindications or side effects and doublechecking dose calculations and prescriptions for errors: 
"I'm always learning new stuff. When you take that responsibility of suddenly putting your name to it, you know, you're always checking - Does that interact with something else? Have I gone through the patient's notes to find every inch of past medical history that might be relevant? It does make you... I think, look at the patient a little bit more holistically and look for every detail, because you know you need to know it all."

\section{Increased credibility and confidence to make recommendations}

Having the prescribing qualification on some occasions increased nurse credibility in relation to other nurses, junior doctors, GPs, physicians and patients. This was important to the nurses who were recommending medication. Having a recognized qualification and demonstrating willingness to take responsibility for prescribing helped to smooth relations between professionals. Other nurses felt that the nurse prescriber's status and credibility was little changed, mainly because they were already highly regarded before becoming a prescriber:

"I think that you are more credible... as a prescriber. I know GPs have been more comfortable with advice coming out of pain clinic because I can put [a prescribing qualification] after my name. Whereas when I started and I didn't have a prescribing qualification, we had a few comments from local GPs to my medical boss saying, 'What makes her qualified to advise me?' And he says it's been much easier to say, 'Well, of course my nurse is qualified to prescribe'." 


\section{DISCUSSION}

The findings of this study indicate that the benefits of nurse prescribing for patients in pain are comparable to those identified in other therapeutic areas in which nurses prescribe medicines. These benefits are in line with those predicted for nurse prescribing (DoH 1999b, 2006), but give deeper insight into the mechanisms by which some of these benefits arise.

In the context of pain, autonomy was defined by the degree to which nurses had control over assessing, diagnosing and making decisions about a patient's medication. Prior to the introduction of nurse prescribing, lack of autonomy hampered practice. Although nurses were able to recommend to doctors that certain medicines should be prescribed, these recommendations were not always followed through. This caused delay and interruption to treatment management. Through prescribing, nurses believed they were able to improve convenience and speed of access to treatment, provide more continuous care, increase efficiency and clinical effectiveness. Autonomy also benefited nurses by improving job satisfaction and making better use of nurses' knowledge and skills. Furthermore, our findings show that the actual process of prescribing can benefit nurses as the continual need to update knowledge generated an accelerated learning process where there was a supportive learning environment.

Through practising autonomously, pain nurses were able to overcome existing inadequacies in the healthcare system, such as limited access to a specialist prescriber. Improvements to safety and quality of care occurred by avoiding or correcting clinically- 
inappropriate prescribing by non-specialists. Indications of poor understanding and sometimes fear of prescribing controlled drugs amongst less experienced healthcare professionals highlights the need for more comprehensive training for a range of these personnel. Specialist pain nurses may have a role to play in that training.

Whilst some of these benefits were reliant on the level of specialist knowledge and expertise nurses had about pain, other benefits were associated with skills considered particular to the nursing profession. The implied superiority of the nurse--patient consultation in terms of communication and information exchange has been consistently reported (Luker et al 1998, Brooks et al 2001, Luker and McHugh 2002, Avery et al 2004, Lewis-Evans and Jester 2004). However, it is when these so called nursing skills meet with the skills and expertise required for prescribing that a grander picture emerges. As reported by Bradley and Nolan (2007), nurses have adopted prescribing alongside more traditional approaches to nursing as a caring role in order to provide more rounded holistic care. It may well be that benefits such as improved concordance, safety and efficient prescribing, arise from combining prescribing expertise with more traditional nursing roles and holistic approaches to health care.

In this study, patient-centred approaches and the principles of concordance were considered especially important when prescribing controlled drugs for patients in pain. The principles of concordance include achieving a partnership between prescriber and patient that enables agreement to be reached over the way in which an illness is understood and treated (Latter 2007b). Nurses believed that their consultation skills, along with their knowledge and expertise, enabled prescribing decisions to be more appropriate to patients' individual needs. Prescriptions were more likely to be effective in 
the first instance and patients were better equipped with the knowledge and understanding needed to adhere to treatment. However, nurse opinions about the effectiveness of their prescribing practice are only part of the story. In research on concordance, Latter (2007b) found that whilst nurse prescribers and their patients thought the principles of concordance were being practised by nurses, they were not always evident to a high degree in observation of practice. Similarly, Latter et al (2007a), in a study of 14 case study sites across the UK, identified that nurse prescribers were inconsistent in enquiring about allergies or use of over-the-counter medication, and did not always record comprehensive details in patient notes. These findings demonstrate the need for further research using a range of outcome measures to study nurse prescribing, rather than relying on self-report methods.

Findings from our broader study highlighted what nurses considered to be the difficulties of nurse prescribing in this area and are reported elsewhere. Relevant to this paper are the importance of a supportive environment, access to suitable education and the indication that barriers such as cost and access to patient records are preventing benefits from occurring for outpatients in chronic pain (Stenner \& Courtenay 2007).

\section{Study limitations}

This was a small study involving self-reported benefits from 26 nurse prescribers who were mainly pain specialists and were mostly working in hospital settings. Further research is needed on the role of generalist nurses prescribing for patients in pain and on the views of doctors, pharmacists and patients. 


\section{CONCLUSION}

There is potential for the benefits of nurse prescribing to be expanded beyond the UK in settings where nurses hold similar roles in the treatment of pain, although further research is recommended to substantiate these findings using a wider range of research methods and outcome measures other than self-report.

\section{Acknowledgements}

This study was undertaken with the help of a research grant from Napp pharmaceuticals. We would like to thank the 'Pain Network' for providing helpful comments on this research. We are grateful to Nicola Carey for help with data collection. 


\section{REFERENCES}

Avery A., Savelyich B. \& Wright L. (2004) Doctors' views on supervising nurse prescribers. Prescriber 5, 56-61.

Bradley E., Campbell P. \& Nolan P. (2005) Nurse prescribers: who are they and how do they perceived their role? Journal of Advanced Nursing 51 (5), 439-448.

Bradley E. \& Nolan P. (2007). Impact of nurse prescribing: a qualitative study. Journal of Advanced Nursing $\mathbf{5 9}$ (2), 120-128.

Brooks N., Otway C., Rashid C., Kilty E. \& Maggs C. (2001) The patient's view: the benefits and limitations of nurse prescribing. British Journal of Community Nursing $\mathbf{6}$ (7), 342-348.

Brown C. A \& Richardson C. (2006) Nurses' in the multi-professional pain team: A study of attitudes, beliefs and treatment endorsements. European Journal of Pain 10, 13-22. Courtenay M., Carey N., and Burke J. (2007) Independent extended and supplementary nurse prescribing practice in the UK: A national questionnaire survey. International Journal of Nursing Studies 44 (7), 1093-1101

Department of Health (1999a) Making a Difference: Strengthening the Nursing, Midwifery and Health Visiting Contribution to Healthcare, Department of Health, London.

Department of Health (1999b) Review of prescribing, supply and administration of medicines. Final report (Crown Report). Department of Health, London.

Department of Health (2000) The NHS Plan: A Plan for Investment, A plan for reform, Department of Health, London.

Department of Health (2002) Supplementary Prescribing for Nurses and Pharmacists 
within the NHS in England, Department of Health, London.

Department of Health (2005) Written Ministerial Statement on the expansion of independent nurse prescribing and introduction of pharmacists independent prescribing, Department of Health, London.

Department of Health (2006) Independent prescribing by Nurses and Pharmacists within the NHS in England. A Guide for Implementation. Department of Health, London.

Hay A., Bradley E., Nolan P. (2004) Supplementary nurse prescribing. Nursing Standard 18 (41), 33-39.

Holloway I. \& Wheeler S. (2002) Qualitative Research in Nursing. Blackwell Publishing, Oxford.

Home Office (2007) Public Consultation: Independent Prescribing of Controlled Drugs by Nurse and Pharmacist Independent Prescribers. Home Office, London.

Johnston B. \& Smith L.N. (2006) Nurses' and patients' perceptions of expert palliative care. Journal of Advanced Nursing 54 (6), 700-709.

Kohr R. \& Sawhney M. (2005) Advanced Practice Nurses' Role in the Treatment of Pain, Canadian Nurse 101 (3), 30-34.

Latter S. \& Courtenay M. (2004) Effectiveness of nurse prescribing: a review of the literature. Journal of Clinical Nursing 13 (1), 26-32.

Latter S., Maben J, Myall M., Courtenay M., Young A. \& Dunn N. (2005) An evaluation of extended formulary independent nurse prescribing. Executive summary of final report, Department of Health, London.

Latter S., Maben J., Myall M., Young A. \& Baileff A. (2007a) Evaluating prescribing competencies and standards used in nurse independent prescribers' prescribing 
consultations: an observational study of practice in England. Journal of Research in Nursing 12 (1), 7-26.

Latter S., Maben J., Myall M. \& Young A. (2007b) Perceptions and practice of concordance in nurses' prescribing consultations: findings from a national questionnaire survey and case studies of practice in England. International Journal of Nursing Studies 44, 9-18.

Lewis-Evans A. \& Jester R. (2004) Nurse prescribers' experiences of prescribing. Journal of Clinical Nursing 13, 796-805.

Luker K.A., Austin L., Hogg C., Ferguson B. \& Smith K. (1997) Patients' views of nurse prescribing. Nursing Times 93 (17), 51-54.

Luker K.A., Austin L., Hogg C., Ferguson B. \& Smith K. (1998) Nurse-patient relationships: the context of nurse prescribing. Journal of Advanced Nursing 28 (2), 235242.

Luker K.A \& McHugh G. (2002) Nurse prescribing from the community nurse's perspective. The International Journal of Pharmacy Practice 10, 273-280.

Nolan P., Sayeed Haque M., Badger F., Dyke R. \& Khan I. (2001) Mental health nurses’ perceptions of nurse prescribing. Journal of Advanced Nursing 36 (4), 527-534. Nursing and Midwifery Council (2007) Number of Independent Extended Nurse Prescribers, Personal communication between second author and NMC February 2007. Patton M.Q. (1990) Qualitative Evaluation and Research Methods (2 ${ }^{\text {nd }}$ ed) Sage, CA

Pellino T. A., Willens J., Polomano R. C. \& Heye M. (2002) The American Society of Pain Management Nurses Practice Analysis: Role Delineation Study. Pain Management 
Nursing 3, (1), 2-15.

Pontin D. \& Jones S. (2007) Children's nurses and nurse prescribing: a case study identifying issues for developing training programmes in the UK. Journal of Clinical Nursing 16, 540-548

Royal College of Anesthetists and the Pain Society (2003) Pain Management

Services: Good Practice. Royal College of Anaesthetists \& The Pain

Society, 1-22 May, London.

Rodden C. (2001) Nurse prescribing: views on autonomy and independence. British

Journal of Community Nursing 6 (7), 350-355

Söderhamn O. \& Idvall E. (2003) Nurses' influence on quality of care in postoperative pain management: A phenomenologcial study. International Journal of Nursing Practice 9, 26-32.

Stenner K. \& Courtenay M. (2007) A qualitative study on the impact of legislation on the prescribing of controlled drugs by nurses. Nurse Prescribing 5 (6), 257-261. 